\title{
Optical Synthesis of Terahertz and Millimeter-Wave Frequencies with Discrete Mode Diode Lasers
}

\author{
Stephen O'Brien $*$ Simon Osborne, David Bitauld, Nicola Brandonisio, and Andreas Amann \\ Tyndall National Institute, University College Cork, Lee Maltings, Cork, Ireland \\ Richard Phelan, Brian Kelly, and James O'Gorman \\ Eblana Photonics, Trinity College Enterprise Centre, Pearse Street, Dublin 2, Ireland
}

\begin{abstract}
It is shown that optical synthesis of terahertz and millimeter-wave frequencies can be achieved using two-mode and mode-locked discrete mode diode lasers. These edge-emitting devices incorporate a spatially varying refractive index profile which is designed according to the spectral output desired of the laser. We first demonstrate a device which supports two primary modes simultaneously with high spectral purity. In this case sinusoidal modulation of the optical intensity at terahertz frequencies can be obtained. Cross saturation of the material gain in quantum well lasers prevents simultaneous lasing of two modes with spacings in the millimeter-wave region. We show finally that by mode-locking of devices that are designed to support a minimal set of four primary modes, we obtain a sinusoidal modulation of the optical intensity in this frequency region.
\end{abstract}

\section{INTRODUCTION}

Synthesis of terahertz $(\mathrm{THz})$ and millimeter-wave (mmWave) frequencies using optoelectronic means is currently the subject of significant interest. Generation of these frequencies in the optical domain can be much less complex than with electrical schemes and also enables signal distribution with the large bandwidth and low losses of optical fibers. Interesting applications are expected in diverse areas including high-bandwidth wireless communication systems, THz imaging and spectroscopy, and radio-astronomy [1, 2].

The most direct route to optical synthesis of these frequencies is simply to mix the outputs of two detuned single mode lasers [5]. This technique is simple to implement and provides very wide tunability. However, because the outputs of the two sources are not correlated, broadening of the linewidth of the generated difference frequency is observed. For many applications, an active feedback mechanism such an optical phase locked loop is necessary to improve the linewidth $[\underline{3}, 4]$.

An alternative for many applications is to electronically modulate the output of a continuous wave single mode laser [6, 7]. Electronics based techniques can combine frequency tunability and narrow linewidth but it is necessary to filter the modes of interest from the intensity modulated spectrum. In addition, higher frequency electronic modulation schemes are correspondingly more complex and expensive to implement.

Two-mode diode lasers represent an interesting alternative approach to the problem on account of their compactness and simplicity. While monolithic two-mode devices do not generally lead to continuous and wide tunability of the difference frequency, there may be significant advantages where the frequency of operation is fixed

*Electronic address: stephen.obrien@tyndall.ie for a particular application. Because the modes share a common cavity, the degradation of the linewidth of the beatnote due to environmental and other factors is expected to be less than in the case of two independent single mode lasers [8]. Many examples of semiconductor lasers that support two modes with a large frequency spacing have been described in the literature. These include external cavity lasers $[9]$, and devices based on distributed Bragg reflections 10 12 and compound cavity designs [13, 14].

Here we demonstrate a powerful approach to mode selection in a Fabry-Perot semiconductor laser that enables optical synthesis of frequencies ranging from $\mathrm{THz}$ to the mmWave region. Depending on the frequency required, two-mode or mode-locked diode lasers that support a minimal set of four pre-selected modes can be used. Our approach relies on a distributed reflection mechanism that is designed as a perturbation of the Fabry-Perot mode spectrum. The cavity geometry is derived directly from an inverse problem solution that has enabled us to design single-mode [15] and two-mode lasers [16] with excellent spectral purity. Single mode devices of this kind were also shown to have very narrow linewidth emission under normal drive conditions [17].

This paper is organised as follows: In section II, we demonstrate that two-mode quantum-well lasers provide a direct route to optical synthesis of $\mathrm{THz}$ frequencies. The stability properties of two-mode devices as a function of the primary mode spacing are also described. It is shown that for primary mode spacings in the mmWave region, cross saturation of the material gain leads to wavelength bistability of the primary modes. In section III, we describe how mode-locking of a device designed to support a minimal set of four primary modes can be used to overcome the problem of wavelength bistability. Finally, prospects for obtaining narrow linewidth $\mathrm{THz}$ and mmWave signals using discrete mode devices are discussed. 


\section{STABILITY PROPERTIES OF TWO-MODE DEVICES AS A FUNCTION OF PRIMARY MODE SPACING}

We have shown that a set of self-consistent equations for the lasing modes can be found by making an expansion about the cavity resonance condition in a FabryPerot laser [16]. The effective index along the FabryPerot cavity is perturbed by $N$ additional features that are described by an index step $\Delta n$. A first order scattering approximation allows Fourier analysis to be used to make a direct connection between the index profile in real space and the threshold gain modulation in wavenumber space. Here we will present measurements of fabricated devices where the index step features are slotted regions etched into the ridge waveguide of the laser. All of the devices considered are multi-quantum well $\mathrm{InP} / \mathrm{InGaAlAs}$ devices with peak emission ranging from $1.3 \mu \mathrm{m}$ to 1.5 $\mu \mathrm{m}$.

Measurements of a two-mode ridge waveguide FabryPerot laser with a peak emission near $1.3 \mu \mathrm{m}$ are shown in Fig. 1 (a). Details of the inverse problem solution and of the design of two-mode diode lasers can be found in [16]. In this case the device length is $350 \mu \mathrm{m}$, and we have chosen a primary mode spacing of four modes which determines a frequency separation of the primary modes of $480 \mathrm{GHz}$. Generally, one finds that as the current is increased from below threshold the primary mode on the short wavelength side reaches threshold first and as the current is increased further the peak power shifts across the primary mode spacing to the long wavelength side. The spectrum shown in Fig. 11 (a) was obtained at a device current of $43.5 \mathrm{~mA}$. At the value of the device current shown the time averaged optical power in each of the primary modes is approximately equal. We refer to this point in the light-current relationship as the twocolor point. One can see that excellent mode selectivity is achieved in this case with the primary mode intensity exceeding the background modes by a factor of $40 \mathrm{~dB}$.

The spectral purity observed in Fig. 11 (a) is limited in this case by power transfer to four-wave mixing sidebands. The presence of these sidebands is significant as they indicate simultaneous lasing of the primary modes. This is confirmed by the intensity autocorrelation measurement shown in the inset of Fig. 1 (a), which confirms a sinusoidal modulation of the intensity output at the primary mode frequency separation of $480 \mathrm{GHz}$. Power spectra of one of the primary modes and of the total intensity are shown in Fig. 1 (b). One can see that there is significant low-frequency antiphase noise in the spectrum of the primary mode, which can be attributed to the global coupling of modes by the carrier density in the device.

These characteristic features of the observed power spectra can be reproduced on the basis of a rate-equation model for the dynamics of the electric fields of each mode $E_{1}$ and $E_{2}$ and for the carrier density $N$. To account for the spontaneous emission noise we add Gaussian white

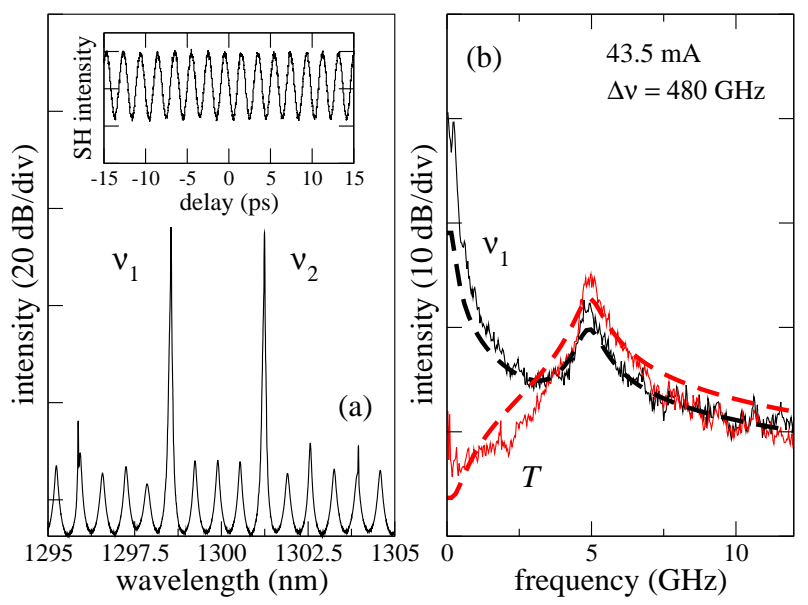

FIG. 1: (a) Optical spectrum of the device at a device current of $43.5 \mathrm{~mA}$ (two-color point). Inset: Background free intensity autocorrelation measurement showing mode beating at $480 \mathrm{GHz}$. (b) Power spectra of one of the primary modes, $\nu_{1}$, and of the total intensity, $T$, measured at the two-color point. The dashed lines are theoretical calculations obtained using equation 5 .

noise terms $\eta_{1}(t)$ and $\eta_{2}(t)$ to the dynamical equations for the fields and arrive at a system of stochastic differential equations of the form

$$
\begin{aligned}
& \dot{E}_{1}=\frac{1}{2}(1+i \alpha)\left(N g_{1}-\gamma\right) E_{1}+\eta_{1} \\
& \dot{E}_{2}=\frac{1}{2}(1+i \alpha)\left(N g_{2}-\gamma\right) E_{2}+\eta_{2} \\
& \dot{N}=J_{0}-\frac{N}{\tau_{s}}-N \sum_{m} g_{m}\left|E_{m}\right|^{2},
\end{aligned}
$$

where the nonlinear modal gain is given by

$$
g_{m}=g_{m}^{(0)}\left(1+\epsilon \sum_{n} \beta_{m n}\left|E_{n}\right|^{2}\right)^{-1} .
$$

In the above equations, $g_{m}^{(0)}=1$ is the linear modal gain. $J_{0}$ is the pump current, $\gamma$ is the cavity decay rate, and $\tau_{s}$ is the carrier lifetime. The phase-amplitude coupling is given by $\alpha$. The parameters $\epsilon \beta_{m n}$ determine the cross and self saturation of the gain. A stable two-mode solution in the free running laser requires $\beta_{m n}<\beta_{m m}$. The Gaussian white noise terms $\eta_{m}(t)$ fulfill the relation $\left\langle\eta_{m}(t) \eta_{n}\left(t^{\prime}\right)\right\rangle=D \delta\left(t-t^{\prime}\right) \delta_{m n}$. A similar model has been successfully used to describe the dynamical features of two-mode lasers with optical injection [18].

Using the standard theory of multivariate OrnsteinUhlenbeck processes [19] we obtain the spectrum matrix in the small noise limit at the two colour point by using the general formula

$$
S(\omega)=\frac{1}{2 \pi}(J+I i \omega)^{-1} \hat{D}\left(J^{T}-I i \omega\right)^{-1} .
$$

Here $J$ is the Jacobian matrix of the system 1 - 3 evaluated at the two-color point and $\hat{D}=\operatorname{diag}(D, D, 0)$ is the noise coupling matrix. The resulting noise spectra for 
the individual modes and for the total output are shown as dashed lines in Fig. 1 (b). One can see that we obtain good qualitative agreement with the corresponding experimental data, including the characteristic feature of the antiphase noise peak at low frequencies of the individual modes and its absence in the total output. The analytical treatment reveals that the origin of the antiphase noise in the dynamics of the individual modes can be traced to one negative real eigenvalue of the Jacobian, whose eigenvector corresponds to fluctuations around the two-color point with a fixed total output and a fixed carrier density. On the other hand, the relaxation oscillation peak at around $5 \mathrm{GHz}$, which is visible in the spectra for the total and the individual modes, is related to a pair of complex conjugated eigenvalues of the Jacobian that describe the exchange of energy between the carriers and the fields. For these calculations the value of $J_{0}$ was fixed at $75 \%$ above its threshold value, $J_{\text {thr. }}$. Other parameters were $\left(\gamma \tau_{s}\right)=800, \epsilon=0.01$, and $\beta \equiv \beta_{m n} / \beta_{m m}=2 / 3$.

Time traces of the total intensity and of one of the primary modes that illustrate the enhanced antiphase noise are shown in Fig. 2 (a). On this scale the total intensity is almost constant, while large fluctuations are visible in the intensity of the primary mode. These intensity fluctuations in the individual modal intensities will lead to increased intensity noise in a generated $\mathrm{THz}$ signal. However, we would like to point out that because the instantaneous frequency of each mode is determined by the dynamics of the carrier density, which in turn depends on the dynamics of the total intensity, antiphase noise does not lead to an additional increase in the phase noise of the $\mathrm{THz}$ signal.

We have found that the level of the intensity noise is strongly dependent on how far the two-color point is located above the device threshold. By varying the substrate temperature, the position of the gain peak can be moved and the two-color point can be brought further from threshold. The normalised standard deviation of the intensity noise is plotted in Fig. 2 (b). One can see that the increased optical power leads to a reduction in the level of intensity noise to less than $5 \%$. A device similar to that shown in Fig. 1 driven at five times threshold was used to generate $\mathrm{THz}$ radiation in a photomixing setup [21].

Because of the increased efficiency of photomixing and applications in high bandwidth wireless communications, the generation of beat notes in the mmWave region around $100 \mathrm{GHz}$ is of significant interest. However, we have found that the simple two-mode device cannot be used in this region because of the phenomenon of wavelength bistability. Wavelength bistability is expected to appear in quantum-well semiconductor lasers once the cross-saturation of the gain exceeds the self-saturation [16]. We have found that this boundary is located in the region of $300 \mathrm{GHz}$. For primary mode spacings less than this, a discontinuous switching of the intensity from one primary mode to the other is observed over a very small current range.
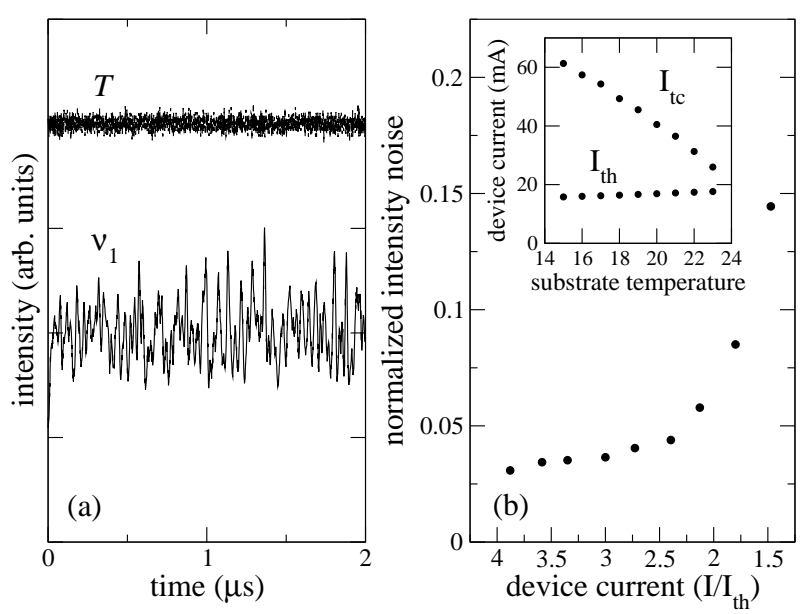

FIG. 2: (a) Intensity time traces of one of the primary modes, $\nu_{1}$, and of the total intensity, $T$, measured at the two-color point. (b) Variation of the normalized standard deviation of the intensity noise in one of the primary modes measured at the two-color point. Inset: Variation of the device current measured at threshold, $\mathrm{I}_{\mathrm{th}}$, and at the two-color point, $\mathrm{I}_{\mathrm{tc}}$, as the substrate temperature is varied.

Optical spectra that illustrate this switching are shown in Fig. 3. These are experimental measurements from a two-mode device with a peak emission near $1490 \mathrm{~nm}$. The primary mode spacing in this case is $250 \mathrm{GHz}$ and one can see that a $20 \mathrm{~dB}$ change in intensity is observed over approximately $1 \mathrm{~mA}$ variation in the device current. In fact, these quasi-single mode states coexist over a small hysteresis region, within which noise driven switching of the intensity between the modes can be observed. This so-called mode-hopping behaviour [22] is associated with a characteristic dwell time, which is the average time measured between switching events. By varying the substrate temperature as before we were able to move the bistability region further from threshold. We found that the corresponding dwell time varied by over four orders of magnitude as shown in Fig. 3 (c). The dashed line in Fig. 3 (c) shows a theoretical calculation of the dwell time, which is obtained by approximating the system 1 3 by a one dimensional stochastic differential equation for the dynamical variable $\phi=\arctan \left(\left|E_{1}\right|^{2} /\left|E_{2}\right|^{2}\right)$, similar to the approach which was taken in [20]. This leads to an Arrhenius formula for the dwell time $\tau_{\mathrm{D}}$ given by

$$
\tau_{\mathrm{D}}=\frac{\sqrt{2} \pi}{\sqrt{e D \gamma \tau_{s} \delta}\left(\hat{P}^{2}-1\right)} \exp \left[\frac{1}{2} \hat{P}^{2}\right]
$$

where $\hat{P}=P \sqrt{\gamma \delta / D \tau_{s}}$ with $P=\frac{1}{2}\left(\frac{J_{0}}{J_{\text {thr }}}-1\right)$ and $\delta=$ $\frac{1}{2} \epsilon(\beta-1)$. For this calculation, we used the same set of parameters as in Fig. 1 (b) apart from the ratio of cross to self saturation which is now given by $\beta=1.1$. The noise strength used for fitting experimental data was $D=9.6 \times 10^{15} \mathrm{~s}^{-2}$. 

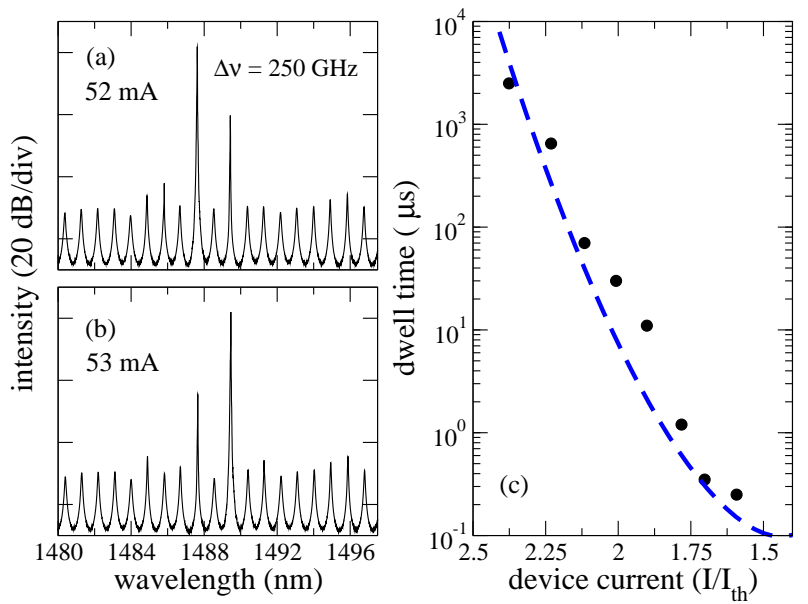

FIG. 3: Optical spectra of the wavelength bistable device before (a) and after (b) the bistability region. (c) Variation of the dwell time in the bistability region as the device current at the bistability point is varied. The dashed line is obtained from the escape time analysis of a reduced one-dimensional model [Eqn. 6].

\section{MILLIMETER-WAVE FREQUENCY SYNTHESIS WITH PASSIVELY MODE-LOCKED DISCRETE MODE LASERS}

In order to avoid the problems associated with wavelength bistability in two-mode quantum-well devices, we have designed passively mode-locked devices that support a minimal set of four primary modes. These devices include a saturable absorber section placed adjacent to one of the cavity mirrors. In the device we consider here, four primary lasing modes are chosen with a spacing of two fundamental modes leading to mode-locking at the first harmonic of the cavity.

The cavity design in this case represents a straightforward extension to four modes of the approach to designing a two-mode device described in [16]. Fig. 4 (a) shows the feature density function which must be sampled in order to define the effective index profile. A schematic picture of the device, high-reflection coated as indicated, is shown in the lower panel of Fig. 4(a). The device length is $545 \mu \mathrm{m}$, and $N=42$ etched features are introduced. The calculated form of the threshold gain spectrum is shown in the inset of Fig. 4 (a).

Experimental measurements of a ridge waveguide Fabry-Perot laser fabricated to the design depicted are shown in Fig. 4 (b). This device has a peak emission near $1.5 \mu \mathrm{m}$ and a saturable absorber section of length $30 \mu \mathrm{m}$ was placed adjacent to the high reflectivity mirror. Fig. 4(b) displays the optical spectrum for a reverse bias voltage of $-2.8 \mathrm{~V}$ applied to the saturable absorber section. The four selected modes are in excess of $20 \mathrm{~dB}$ stronger than all other modes and have a frequency separation of $160 \mathrm{GHz}$. Because most of the laser light is concentrated in the two central primary modes, the resulting time evolution of the power is essentially a beating be-

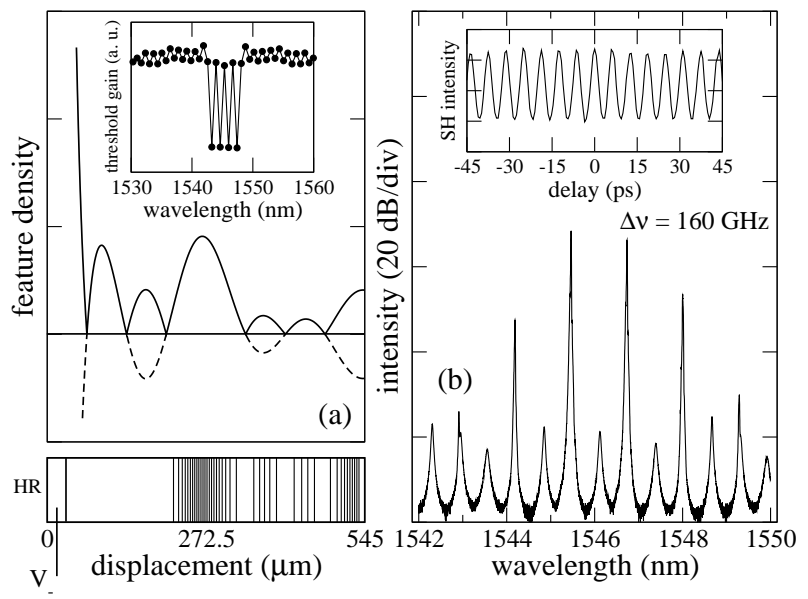

FIG. 4: (a) Feature density function (solid line). Dashed lines indicate intervals where the Fourier transform of the spectral filtering function chosen is negative. Inset: Calculation of the threshold gain of modes for the laser cavity schematically pictured in the lower panel of the figure. Lower panel: Laser cavity schematic indicating the locations of the additional features. The device is high-reflection (HR) coated and includes a saturable absorber section as indicated. (b) Optical spectrum of the device at a device current of $110 \mathrm{~mA}$ and a reverse bias voltage of $-2.8 \mathrm{~V}$. Inset: Background free intensity autocorrelation measurement showing mode beating at $160 \mathrm{GHz}$.

tween these two modes. The inset of Fig. 4 (b) displays the intensity autocorrelation which confirms a sinusoidal intensity modulation at the mode-locking frequency.

We note that while examples of mode-locked diode lasers with a sinusoidal intensity modulation have been presented in the literature [13], the sinusoidal modulation can be attributed to the fact that the primary mode spacing is comparable to the gain bandwidth in these devices. For narrower mode spacings a mode locked device will generally oscillate on many modes generating pulsed output. External filtering of pairs of modes from the mode-locked spectrum is then necessary to obtain sinusoidal output [23]. Our approach to this problem can be regarded as integrating the functions of mode-locking and spectral filtering in a single device.

These results demonstrate that discrete mode devices provide a convenient means to realize $\mathrm{THz}$ and mmWave frequencies in the optical domain. Microwave frequency synthesis will also be possible in longer devices with a correspondingly narrower mode spacing. We are currently investigating the dependence of the linewidth of the beatnote on the drive parameters. Preliminary measurements indicate that in the mode-locked device, this linewidth is in the region of $5 \mathrm{MHz}$. It should be noted however that because the mode selection mechanism in our device acts to predetermine the carrier wave frequency in the modelocked state, we expect that the characteristics of this state will also depend on where the central mode frequency is placed with respect to the material gain and loss dispersion. 
While mode-locking was achieved at $160 \mathrm{GHz}$ in the current device, results suggest that quantum-well material can be used for mode-locking frequencies as large as 1 $\mathrm{THz}$ [24]. However, the use of self-assembled gain materials may provide significant advantages such as reduced amplified spontaneous emission noise, faster carrier recovery and limited alpha factors that can further improve the stability of the mode-locked state. The possibilities suggested by techniques such as hybrid mode-locking are also interesting in this regard. Significantly, single-mode discrete mode devices have been shown to have optical linewidths as small as $100 \mathrm{kHz}$ under normal drive conditions [17]. This suggests that a mode beating linewidth in the sub-MHz range should be achievable with discrete mode devices.

\section{CONCLUSION}

We have demonstrated that discrete mode diode lasers provide a direct route to optical synthesis of $\mathrm{THz}$ and mmWave frequencies. These devices are specially designed multimode semiconductor lasers with a predefined number and spacing of primary lasing modes. For frequency separations in the $\mathrm{THz}$ region, it was shown that a single section quantum-well device can support two optical modes simultaneously with high spectral purity. We showed that wavelength bistability appears for mode spacings less than approximately $300 \mathrm{GHz}$ and that this phenomenon prevents simultaneous lasing of two modes. Finally, we showed that passive mode-locking of devices that support only four primary modes can be used to overcome the difficulties presented by wavelength bistability. In this case sinusoidal modulation of the optical intensity at mmWave frequencies was achieved.
[1] J. Yao, "Microwave photonics," J. Lightwave Tech., vol. 27, no. 3, pp. 314-335, Feb. 2009.

[2] P. H. Siegel, "Terahertz technology," IEEE Trans. Microwave Theory Tech., vol. 50, no. 3, pp. 910-928, Mar. 2002.

[3] R. C. Steele, "Optical phase-locked loop using semiconductor laser diodes," IEE Electron. Lett., vol. 19, no. 2, pp. 69-71, Jan. 1983.

[4] F. Freiderich, G. Schuricht, A. Deninger, F. Lison, G. Spickermann, P. H. Bolívar, and H. G. Roskos, "Phase locking of the beat signal of two distributed-feedback diode lasers to oscillators working in the $\mathrm{MHz}$ to $\mathrm{THz}$ range," Opt. Express, vol. 18, no. 8, pp. 8621-8629, Apr. 2010 .

[5] K. A. McIntosh, E. R. Brown, K. B. Nichols, O. B. McMahon, W. F. DiNatale, and T. M. Lyszczarz, "Terahertz photomixing with diode lasers in lowtemperature-grown GaAs," Appl. Phys. Lett., vol. 67, no. 26, pp. 3844-3846, Dec. 1995.

[6] A. Hirata, M. Harada, and T. Nagatsuma, "120-GHz wireless link using photonic techniques for generation, modulation, and emission of millimter wave signals," J. Lightwave Tech., vol. 21, no. 10, pp. 2145-2153, Oct. 2003.

[7] S. Fukushima, C. F. C. Silva, Y. Muramoto, and A. J. Seeds, "Optoelectronic millimeter-wave synthesis using an optical frequency comb generator, optically injection locked lasers, and a unitraveling-carrier photodiode," J. Lightwave Tech., vol. 21, no. 12, pp. 3043-3051, Dec. 2003.

[8] M. Tani, O. Morikawa, S. Matsuura, and M. Hangyo, "Generation of terahertz radiation by photomixing with dual- and multiple-mode lasers," Semicond. Sci. Technol., vol. 20, no. 7, pp. 151-163, Jul. 2005.

[9] I. Park, I. Fischer and W. Elsäßer, "Highly nondegenerate four-wave mixing in a tunable dual-mode semicon-

\section{Acknowledgment}

The authors thank Science Foundation Ireland and Enterprise Ireland for financial support of this work. ductor laser," Appl. Phys. Lett., vol. 84, no. 25, pp. 51895191, Jun. 2004.

[10] S. Iio, M. Suehiro, T. Hirata, and T. Hidaka, "Twolongitudinal-mode laser diodes," IEEE Photon. Tech. Lett., vol. 7, no. 9, pp. 959-961, Sep. 1995.

[11] Y. Matsui, M. D. Pelusi, S. Arahira, and Y. Ogawa, "Beat frequency generation up to $3.4 \mathrm{THz}$ from simultaneous two-mode lasing operation of sampled-grating DBR laser," IEE Electron. Lett., vol. 35, no. 6, pp. 472474, Mar. 2008.

[12] S. D. Roh, T. S. Yeoh, R. B. Swint, A. E. Huber, J. S. Woo, J. J. Coleman, "Dual-wavelength InGaAsGaAs ridge waveguide distributed Bragg reflector lasers with tunable mode separation," IEEE Photon. Technol. Lett., vol. 12, no. 10, pp. 1307-1309, Oct. 2000.

[13] D. A. Yanson, M. W. Street, S.D. McDougall, I. G. Thayne, J. H. Marsh, and E. A. Avrutin, "Ultrafast harmonic mode-locking of monolithic compound-cavity laser diodes incorporating photonic-bandgap reflectors," IEEE J. Quantum Electron., vol. 38, no. 1, pp. 1-11, Jan. 2002.

[14] N. Kim, J. Shin, E. Sim, C. W. Lee, D.-S. Yee, M. Y. Jeon, Y. Jang, and K. H. Park, "Monolithic dualmode distributed feedback semiconductor laser for tunable continuous-wave terahertz generation," Opt. Express, vol. 17, no. 16, pp. 13851-13859, Jul. 2009.

[15] S. O'Brien and E. P. O'Reilly, "Theory of improved spectral purity in index patterned Fabry-Perot lasers," Appl. Phys. Lett., vol. 86, no. 20, 201101, May 2005.

[16] S. O'Brien, S. Osborne, K. Buckley, R. Fehse, A. Amann, E. P. O'Reilly, L. P. Barry, P. Anandarajah, J. Patchell, and J. O'Gorman, "Inverse scattering approach to multiwavelength Fabry-Perot laser design," Phys. Rev. A, vol. 74, no. 6, 063814, Dec. 2006.

[17] B. Kelly, R. Phelan, D. Jones, C. Herbert, J. O'Carroll, M. Rensing, J. Wendelboe, C. B. Watts, A. Kaszubowska-Anandarajah, P. Perry, C. Guignard, 
L. P. Barry, and J. O'Gorman, "Discrete mode laser diodes with very narrow linewidth emission," IEE Electron. Lett., vol. 43, no. 23, pp. 1282-1284, Nov. 2007.

[18] S. Osborne, A. Amann, K. Buckley, G. Ryan, S. P. Hegarty, G. Huyet, and S. O'Brien, "Antiphase dynamics in a multimode semiconductor laser with optical injection," Phys. Rev. A, vol. 79, no. 2, 023834, Feb. 2009.

[19] C. Gardiner, Stochastic Methods, Springer, Berlin, 2009.

[20] F. Pedaci, S. Lepri, S. Balle, G. Giacomelli, M. Giudici, and J. R. Tredicce, "Multiplicative noise in the longitudinal mode dynamics of a bulk semiconductor laser," Phys. Rev. E, vol. 73, no. 4, 041101, Apr. 2006.

[21] S. Osborne, S. O'Brien, E. P. O'Reilly, P. G. Huggard, and B. N. Ellison, "Generation of $\mathrm{CW} 0.5 \mathrm{THz}$ radia- tion by photomixing the output of a two-colour $1.49 \mu \mathrm{m}$ Fabry-Perot laser diode," IEE Electron. Lett., vol. 44, no. 4, pp. 296-297, Feb. 2008.

[22] M. Ohtsu and Y. Teramachi, "Analyses of mode partition and mode hopping in semiconductor lasers," IEEE J. Quantum Electron., vol. 25, no. 1, pp. 31-38, Jan. 1989.

[23] M. D. Pelusi, H. F. Liu, D. Novak, and Y. Ogawa, "THz optical beat frequency generation from a single mode locked semiconductor laser," Appl. Phys. Lett., vol. 71, no. 4, pp. 449-451, Jul. 1997.

[24] S. Arahira, S. Oshiba, Y. Matsui, T. Kunii, and Y. Ogawa, "Terahertz-rate optical pulse generation from a passively mode-locked semiconductor laser diode," Optics Lett., vol. 19, no. 11, pp. 834-836, Jun. 1994. 